\title{
Review
}

\section{Molecular mechanisms of excitotoxicity and their relevance to pathogenesis of neurodegenerative diseases}

\author{
Xiao-xia DONG, Yan WANG, Zheng-hong QIN* \\ Department of Pharmacology and Laboratory of Aging and Nervous Diseases, Soochow University School of Medicine, Suzhou 215123, China
}

\begin{abstract}
A pivotal role for excitotoxicity in neurodegenerative diseases is gaining increasingly more acceptance, but the underlying mechanisms through which it participates in neurodegeneration still need further investigation. Excessive activation of glutamate receptors by excitatory amino acids leads to a number of deleterious consequences, including impairment of calcium buffering, generation of free radicals, activation of the mitochondrial permeability transition and secondary excitotoxicity. Recent studies implicate excitotoxicity in a variety of neuropathological conditions, suggesting that neurodegenerative diseases with distinct genetic etiologies may share excitotoxicity as a common pathogenic pathway. Thus, understanding the pathways involved in excitotoxicity is of critical importance for the future clinical treatment of many neurodegenerative diseases. This review discusses the current understanding of excitotoxic mechanisms and how they are involved in the pathogenesis of neurodegenerative diseases.
\end{abstract}

Keywords: excitotoxicity; glutamate receptors; mitochondria; calcium; oxidative stress; neurodegenerative diseases

Acta Pharmacologica Sinica (2009) 30: 379-387; doi: 10.1038/aps.2009.24

\section{Introduction}

The increase in human lifespan observed in industrialized countries has been accompanied by a marked prevalence of neurodegenerative diseases. These diseases are preferential lines of study in gerontology and geriatrics since healthy aging is the primary concern in those fields. Human neurodegenerative diseases are characterized by progressive dysfunction and loss of neurons induced by particular neurological deficits ${ }^{[1]}$. Over the past several years, research has demonstrated that excitatory amino acids serve as the major excitatory neurotransmitters in the cerebral cortex and hippocampus. Neurons that contain excitatory amino acids play crucial roles in psychological functions such as learning and memory. Disturbances of the excitatory amino acid system may contribute to the pathogenesis of schizophrenia and other neuropsychiatric syndromes associated with delirium or dementia ${ }^{[2]}$. On the other hand, overactivity of the excitatory amino acid system is also harmful. Excitotoxicity is defined as cell death resulting from the toxic actions of excitatory amino acids. Because glutamate is the major excitatory

* Correspondence to Prof Zheng-hong QIN.

E-mail zhqin5@hotmail.com

Received 2008-12-04 Accepted 2009-02-19 neurotransmitter in the mammalian central nervous system (CNS), neuronal excitotoxicity usually refers to the injury and death of neurons arising from prolonged exposure to glutamate and the associated excessive influx of ions into the cell. The resulting calcium overload is particularly neurotoxic, leading to the activation of enzymes that degrade proteins, membranes and nucleic acids ${ }^{[3]}$.

Neurodegenerative diseases are a heterogeneous group of illnesses with distinct clinical phenotypes and genetic etiologies. Although most neurodegenerative diseases are sporadic - that is, no genetic linkage is known - others can be traced to specific genetic mutations. Although these mutations affect a wide variety of proteins, substantial evidence points to excitotoxicity as a fundamental mechanism involved in neuronal degeneration. Overactivation of glutamate receptors impairs cellular calcium homeostasis and activates nitric oxide synthesis, generation of free radicals and programmed cell death ${ }^{[4]}$. As reviewed in the following sections, many studies have provided some support for each effect, although the roles of these molecules are still incompletely defined. This review will focus on cellular signaling cascades triggered by excitotoxic insult in neurodegenerative diseases. Identification of key signaling events that contribute to excitotoxic cell death will provide not only insights 
into the molecular basis of how neuronal cells die, but also potential approaches for therapeutic intervention targeting excitotoxic signaling pathways in neurodegenerative disorders.

\section{Ionotropic and metabotropic glutamate receptors}

Glutamatergic neurons form the main excitatory system in the brain and play a pivotal role in many neurophysiological functions. Under normal conditions, glutamate is the major neurotransmitter in primary perception and cognition in the brain, producing an excitatory response. This response is generated following an interaction of glutamate with receptors composing cation channels. Excessive activation of glutamate receptors can result in neuronal dysfunction and death, a process called excitotoxicity ${ }^{[5]}$. There is an excess of glutamate and glutamatergic activity in certain neurodegenerative diseases. The excitatory effects of glutamate are exerted via the activation of three major types of ionotropic receptors and several classes of metabotropic receptors linked to G-proteins. The major ionotropic receptors activated by glutamate are commonly referred to as the $N$-methyl-D-aspartic acid (NMDA), $\alpha$-amino-3-hydroxy-5methylisoxazole-4-propionate (AMPA) and kainic acid (KA) receptors. These ionotropic receptors are ligand-gated ion channels permeable to various cations ${ }^{[6]}$.

NMDA receptors are $\mathrm{Ca}^{2+}$-favoring glutamate-gated ion channels that are expressed in most central neurons and were initially held responsible for neuronal injury, owing to their high $\mathrm{Ca}^{2+}$ permeability and conductance properties ${ }^{[7]}$. Continuous activation of large numbers of NMDA receptors (especially the NR1/NR2B-subtype) leads to increases in intracellular calcium loads and catabolic enzyme activities, which can trigger a cascade of events eventually leading to apoptosis or necrosis ${ }^{[8]}$. These downstream effects include mitochondrial membrane depolarization, caspase activation, production of toxic oxygen and nitrogen free radicals, and cellular toxicity ${ }^{[9,10]}$. The NMDA receptors are also effective in mediating excitotoxic neuronal injury. For example, neurons expressing high levels of NMDA receptors are lost early in the striatum of individuals affected with neurodegenerative disease, and injection of NMDA receptor agonists into the striatum of rodents or non-human primates recapitulates the pattern of neuronal damage in Huntington's disease $(\mathrm{HD})^{[11]}$.

AMPA-type glutamate receptors have also been implicated in excitotoxicity because assemblies of these receptors are highly permeable to $\mathrm{Ca}^{2+}$ and possibly contribute to the delayed neuronal cell death processes induced by $\mathrm{Ca}^{2+}$ overload. The $\mathrm{Ca}^{2+}$ permeability of the AMPA receptor is determined by the presence or absence of the GluR2 subunit in the receptor complex. Low expression of GluR2 permits the construction of AMPA receptors with high $\mathrm{Ca}^{2+}$ permeability and contributes to neuronal degeneration in ischemia. Surprisingly, decreasing GluR2 levels or selective blockage of $\mathrm{Ca}^{2+}$-permeable AMPA receptors was also shown to protect against neurodegeneration $^{[12]}$. However, many additional studies have demonstrated that changes in glutamate receptor expression after neurological insults may not be so selective. Therefore, under pathological conditions, such as seizures induced by kainate (KA, a potent agonist of the AMPA/KA class of glutamate receptors)- or hypoxia-ischemia, many principal cells may experience increased $\mathrm{Ca}^{2+}$ influx, regardless of the existing stoichiometry of AMPA or NMDA receptor assemblies ${ }^{[13]}$. Several recent studies suggest that excessive stimulation of non-NMDA glutamate receptors with KA can induce autophagy and activation of lysosomal enzymes. The autophagy/lysosome pathway, in turn, plays an important role in excitotoxic neuronal injury ${ }^{[14,15]}$.

In neurodegenerative diseases, metabotropic (mGluR) receptors mediate slow synaptic responses, owing to their coupling with intracellular G-proteins. For example, the mGluR1 and mGluR5 subunit subtypes, which exist in a number of alternatively spliced forms, are coupled to the inositol trisphosphate (IP3)/ $\mathrm{Ca}^{2+}$ signal transduction pathway and can thus affect protein kinase activation and stimulation of $\mathrm{Ca}^{2+}$ release from neuronal stores, both of which can trigger delayed cell death processes ${ }^{[16]}$. On the other hand, mGluR2 may help to mediate the survival of neurons in the face of selective neuronal dysfunction and degeneration in Alzheimer's disease (AD). Activation of mGluR2 increases the phosphorylation of tau and reduces oxidative stressmediated cytotoxicity in neuronal cells ${ }^{[17]}$.

Many lines of evidence demonstrate an increase in glutamate or other endogenous glutamatergic agonists in neurodegenerative diseases. Considerable evidence indicates that an excitotoxic response arising from increased extracellular glutamate is likely to be important in determining the extent of tissue damage ${ }^{[18]}$. An increase in glutamate receptor activity could induce proapoptotic proteins such as $\mathrm{p} 53$, leading to neuronal injury and death through apoptosis and autophagy $^{[19]}$.

\section{Excitotoxicity and ions $\left(\mathrm{Na}^{+}, \mathrm{Cl}^{-}, \mathrm{Ca}^{2+}\right)$}

Acute excitotoxicity is thought to be mediated by excessive depolarization of the postsynaptic membrane. This results in an osmotic imbalance when countered by an influx 
of $\mathrm{Na}^{+}, \mathrm{Cl}^{-}$, and water, leading to the eventual rupture of cell membranes ${ }^{[20]}$. Numerous reports indicate that acute excitotoxic neurodegeneration following glutamate receptor activation is dependent on $\mathrm{Na}^{+}$and $\mathrm{Cl}^{-}$entry. Accordingly, removal of extracellular $\mathrm{Na}^{+}$or $\mathrm{Cl}^{-}$abolishes NMDA-mediated neurodegeneration ${ }^{[21]}$.

A potential role for $\mathrm{Cl}^{-}$ion transporters in neuronal excitotoxicity has been investigated. $\mathrm{Cl}^{-}$movement is a central component of the acute excitotoxic response in neurons ${ }^{[22]}$. A significant increase in the intracellular $\mathrm{Cl}^{-}$concentration is observed in hippocampal neurons during neurotoxicity induced by glutamate and oxygen/glucose deprivation $(\mathrm{OGD})^{[23]}$. Some studies suggest that cation-dependent $\mathrm{Cl}^{-}$ transport protein $\mathrm{Na}-\mathrm{K}-\mathrm{Cl}$ cotransporter type 1 (NKCC1) is involved in the initial stages of cell damage that depend on extracellular $\mathrm{Na}^{+}$and $\mathrm{Cl}^{-}$. Many results imply that NKCC1 contributes to ischemic neuronal damage by facilitating excessive $\mathrm{Na}^{+}$and $\mathrm{Cl}^{-}$entry during the NMDA-mediated excitotoxicity. Inhibition of NKCC1 activity reduces NMDA-induced swelling and glutamate-mediated neurotoxicity and significantly attenuates OGD-induced neuronal death $^{[24]}$. In addition, a substantial number of studies using neuronal cells have reported that excitotoxic glutamate increases the influx of extracellular $\mathrm{Ca}^{2+}$, resulting in intracellular acidification, which stimulates several $\mathrm{pH}$-regulating systems, including the $\mathrm{Na}^{+}-\mathrm{H}^{+}$exchanger (NHE). Activation of NHE leads to a decrease in the activity of the $\mathrm{Na}^{+}-\mathrm{Ca}^{2+}$ exchanger. Because intracellular $\mathrm{Ca}^{2+}$ elevation can exacerbate the process of cell death, inhibition of NHE has been proposed to attenuate ischemia-induced cell death in various cells $^{[25]}$.

Sustained $\mathrm{Ca}^{2+}$ influx through glutamate receptor channels is thought to represent a common pathway of neuronal cell death. Excess levels of glutamate in the CNS can result in elevated intracellular $\mathrm{Ca}^{2+}$ levels, which in turn cause a rise in the $\mathrm{Ca}^{2+}$ concentration in sensitive organelles such as mitochondria and the endoplasmic reticulum ${ }^{[16]}$. Because of the essential role of $\mathrm{Ca}^{2+}$ in promoting cell death, blocking $\mathrm{Ca}^{2+}$ flux from the endoplasmic reticulum to the mitochondria or buffering intracellular $\mathrm{Ca}^{2+}$ can reduce cellular sensitivity to apoptotic stimuli ${ }^{[25]}$. The NMDA-mediated excessive $\mathrm{Ca}^{2+}$ entry into the cytosol activates calcineurin, which induces apoptosis in both rat hippocampal neurons and stable cell lines such as HeLa cells ${ }^{[26-28]}$. Additionally, a number of studies have shown that activation of calpain, a calciumdependent apoptotic protease, also precedes cell death under various conditions of elevated cytosolic $\mathrm{Ca}^{2+[29,30]}$.

Notably, however, large increases in intracellular $\mathrm{Ca}^{2+}$ can also suppress cell-death genes and prevent programmed cell death, instead promoting neuronal survival. This effect is reportedly mediated by sustained elevation of cytoplasmic free $\mathrm{Ca}^{2+}$ concentrations caused by influx of $\mathrm{Ca}^{2+}$ through voltage-gated channels activated by $\mathrm{K}^{+}$-induced chronic depolarization ${ }^{[31]}$. However, a failure to restore $\mathrm{Ca}^{2+}$ homeostasis would still lead to calcium-mediated neurotoxicity and cell death in the excitotoxicity hypothesis.

\section{Excitotoxicity and oxidative stress}

Oxidative stress is a major player in the pathology of neurodegenerative disorders. The relationship between oxidative stress and neuronal death has been extensively investigated. Oxidative stress damages nucleic acids, proteins and lipids and potentially opens the mitochondrial permeability transition pore, which in turn can further stimulate ROS production, worsen energy failure and release proapoptotic factors such as cytochrome $c$ into the cytoplasm ${ }^{[32]}$. Generation of high levels of ROS and downregulation of anti-oxidant mechanisms result in neuronal cell death in neurodegenerative diseases ${ }^{[33]}$.

Free radicals are referred to as oxidizing agents. Humans are constantly exposed to free radicals created by internal cellular metabolic processes ${ }^{[34]}$. The most common cellular free radicals are superoxide radical $\left(\mathrm{O}_{2^{\cdot-}}\right)$, peroxynitrite $\left(\mathrm{ONOO}^{-}\right)$and hydroxyl radical $(\mathrm{OH} \cdot)$; the latter two species are potentially harmful after hemolytic scission due to the generation of the reactive hydroxyl radical. When antioxidant systems become overwhelmed by these free radicals, oxidative damage and cell death can occur. Free radicals can also damage proteins and nucleic acids, leading to cell death by necrosis or apoptosis ${ }^{[35]}$. Cells normally have a number of mechanisms by which they defend against damage induced by free radicals. Problems occur when production of ROS exceeds their elimination by the antioxidant protection systems or when the latter are damaged. This imbalance between cellular production of ROS and the inability of cells to defend against their effects is called oxidative stress, which is a major factor in the pathogenesis of neuronal damage and is involved in acute and chronic CNS injury ${ }^{[36]}$. In addition, an important mechanism of $\mathrm{O}_{2} \cdot-$ toxicity is its direct oxidation and resulting inactivation of iron-sulfur $(\mathrm{Fe}-\mathrm{S})$ proteins, leading to the release of iron. Although oxidative inactivation of $\mathrm{Fe}-\mathrm{S}$ proteins is known to underlie $\mathrm{O}_{2} \cdot-$ toxicity in bacteria and yeast ${ }^{[37]}$, whether this mechanism contributes to injury in the mammalian brain is unknown and will thus be the focus of future studies.

Nitric oxide (NO) production increases in neurodegenerative diseases as a consequence of oxidative stress. In 
addition to regulating cerebral vasoactivity, NO possesses various physiological roles. NO synthesis is activated in cerebrovascular disease by the release of glutamate combined with inhibition of glutamate removal, which leads to NMDA receptor overactivation and excess $\mathrm{Ca}^{2+}$ influx ${ }^{[38]}$. It is believed that the toxic effects of $\mathrm{NO}$ result from the actions of its downstream metabolite, ONOO-, according to models implicating $\mathrm{NO}$ in neurodegeneration. ONOO- is a highly reactive oxidant formed when NO reacts with superoxide radicals, which also regulate excitotoxicity and induce oxidative DNA damage ${ }^{[39]}$. Evidence suggests that in $\mathrm{AD}$, ONOO- can both promote DNA fragmentation by oxidative damage and prevent protein phosphorylation by tyrosine nitration, therefore disturbing signal transduction mediated by tyrosine kinases ${ }^{[40]}$. Recently, it was shown that NO induces the overexpression of metalloproteinases, which in turn destroy the environment that surrounds neuronal cells. The extracellular proteolytic cascades that are triggered by metalloproteinase can disrupt the extracellular matrix, contribute to cell detachment and lead to anoikis (apoptosis due to cell detachment from the substrate $)^{[41]}$. Thus, the clinical convergence of advanced aging with the presence of $\mathrm{NO}$ and ONOO-can exacerbate the neuronal damage characteristic of neurodegenerative disease patients.

ROS are free radicals that are normal products of oxygen metabolism and are produced in excess during the course of ischemia/reperfusion through a variety of mechanisms. Intracellular ROS are capable of inducing damage and, in severe cases, cell death through mitochondrial alterations leading to the release of cytochrome $\mathrm{c}^{[42,43]}$ through activation of the JNK pathway ${ }^{[44]}$ or by activation of nuclear factor- $\kappa B$ (NF- $\kappa B)$ transcription factors ${ }^{[45]}$. The ability to control ROS is thus critical in neurodegenerative diseases, because neuronal damage occurs when the "oxidant- antioxidant" balance is disturbed in favor of excess oxidative stress $^{[46]}$. A recent study suggests that a ROS-scavenger effectively protected human neuroglioma against both necrotic and apoptotic cell death induced by hydrogen peroxide ${ }^{[4]]}$.

\section{Excitotoxicity and mitochondria-mediated apoptosis and autophagy}

Mitochondria represent the energy powerhouses and buffering sinks of the cell. Mitochondria not only function as the site of oxidative phosphorylation and cellular respiration, but also play a critical role in maintaining a low concentration of calcium in the cytosol. Changes in either of these critical functions of mitochondria have formidable consequences and often determine the cell's fate in survival/ death signaling pathways. In particular, excessive uptake of calcium or generation of ROS induces activation of the mitochondrial permeability transition and subsequent release of calcium and proapoptotic factors into the cytosol ${ }^{[48,49]}$.

There is evidence that mitochondrial dysfunction and impairment of respiratory complexes play a role in the neuronal loss experienced in neurodegenerative diseases. Recently, the mitochondrion has come to be considered a pivotal organelle in determining cell fate, because it may act as an 'on-off' switch modulating autophagy and apoptosis. Diverse autophagic or apoptotic signals may converge on mitochondria and provoke the permeability transition that results in release of apoptogenic proteins into the cytosol, where they trigger caspase-dependent apoptosis or promote autophagy. These factors are thought to regulate cell death by degrading cytoskeletal proteins, as well as those involved in DNA repair, and by activating DNases ${ }^{[50]}$. Low-intensity stress can cause depolarization of mitochondria during the permeability transition, leading to the induction of autophagy, which in turn selectively removes damaged mitochondria as a cytoprotective mechanism ${ }^{[51]}$. An overburdened autophagic apparatus may release lysosomal enzymes and possibly other factors in order to promote cell death ${ }^{[15]}$. In addition to the processes mentioned above, several intracellular signals, including DNA damage and endoplasmic reticulum stress, can also induce mitochondrial membrane permeabilization $^{[52]}$ to release proapoptotic factors from the intermembrane space.

We found that quinolinate- or KA-induced apoptosis is preceded by a substantial rise in NF-kB binding activity in striatal nuclear extracts. Pretreatment with a cell-permeable recombinant peptide inhibitor of NF- $\mathrm{kB}$ selectively blocked quinolinate-induced NF- $\mathrm{kB}$ nuclear translocation as well as apoptosis ${ }^{[33]}$. We have also proposed that certain neuroprotective effects of diverse agents may reduce excitotoxic neuronal injury by inhibiting activation of the NF- $\mathrm{kB}$ cascade ${ }^{[54]}$. In the NMDA-triggered apoptotic process involving NF- $\mathrm{kB}$ activation, NF- $\mathrm{kB}$ regulates the expression of many proteins, including $\mathrm{c}-\mathrm{Myc}$ and $\mathrm{p} 53$, which in turn regulate a broad range of physiological and pathological responses ${ }^{[55,56]}$. Levels of both proteins increase upon NF-kB nuclear translocation and play prominent roles in the control of the cell cycle and apoptosis in dividing cells ${ }^{[57]}$. Moreover, p53 binding sites were identified on the rat catalase promoter and confirmed in vivo, and p53 knockdown resulted in an increase in basal cellular ROS levels and increased susceptibility to oxidative cell death ${ }^{[58]}$.

Neuronal mitochondria have a high capacity to store calcium ions and can therefore protect neurons against transient 
elevations in intracellular calcium concentrations during neuronal hyperactivity ${ }^{[59]}$. Under inflammatory conditions or with increased levels of ions, macrophages, astrocytes, and endothelial cells produce excess NO, potentially leading to oxidative stress. NO is considered the chemical species that drives tissue dysfunction in inflammation, neurological diseases, and aging ${ }^{[60]}$. Therefore, mitochondrial dysfunction can induce cell death by affecting cellular calcium homeostasis, enhancing free radical generation, or releasing apoptogenic proteins. It is expected that prevention or delaying of mitochondrial dysfunction upon aging will decrease the neurological deficits experienced by elderly people.

\section{Excitotoxicity in neurodegenerative diseases}

Excitotoxicity in Huntington's disease Huntington's disease (HD) is an inherited neurodegenerative disorder that affects cognition, motor function and mood. Neuropsychiatry changes are caused by the dysfunction or death of specific neuronal cell types in the brain. GABAergic projections of medium-size spiny neurons (MSNs) of the neostriatum are the most severely affected ${ }^{[61]}$. Notably, increasing GABA receptor function has been shown to promote neuronal survival after ischemia by depressing the overall excitability of the cell ${ }^{[62,63]}$. The HD gene and its protein product, huntingtin (htt), were identified over a decade ago, and in recent years many investigations have attempted to reveal the pathogenic mechanisms underlying development of the disease. Researchers have been most successful in identifying the molecular pathways leading to HD pathogenesis, including changes in electrophysiological properties and glutamate receptor sensitivity in cells expressing polyQ-expanded $\mathrm{htt}{ }^{[64]}$.

One of the hypotheses that has been put forward to explain the exquisite sensitivity of the medium spiny projection neurons of the striatum to HD is the "excitotoxicity hypothesis." In the context of HD, the mutant htt protein forms insoluble nuclear aggregates that have been proposed to play a key role in neuronal cell death. Other investigators suggest that expression of mutant htt enhances activity of NMDA receptors and disturbs calcium signaling ${ }^{[65]}$. Some reports have indicated that the post-synaptic density protein PSD-95 may act as a link between htt and NMDAR function ${ }^{[66]}$. PSD-95 itself has been shown to bind the C-terminal tail of NR2 subunits and helps to stabilize NMDARs in cell surface clusters ${ }^{[67]}$. Htt was associated with PSD-95 in transfected 293T cells, and co-immunoprecipitation studies in human cortical tissue revealed interactions of htt with both PSD-95 and NMDAR subunits NR1, NR2A and NR2B ${ }^{[68]}$, suggesting that htt may associate with NMDARs via PSD-95. Recent research has shown that the polyQ expansion in htt is associated with an increase in NR1A/NR2B-mediated excitotoxic cell death. Full-length mutant htt enhances expression of NR1A/NR2B, which is the predominant NMDAR subtype in neostriatal medium-size spiny neurons ${ }^{[69]}$. This relationship may explain the sensitivity of this cell type, if they do indeed die as a result of NMDA-induced apoptosis.

Some investigators have found that overstimulation of NMDA or AMPA-type glutamate receptors can induce apoptosis in projection neurons in vitro and in vivo, but some antiapoptotic proteins in interneurons may confer neuroprotection against excitotoxic challenge, offering an additional explanation for the better survival of striatal interneurons in patients with $\mathrm{HD}$ as well as in excitotoxic models of this disease $^{[70]}$. Altered NMDAR function has been reported in corticostriatal synapses, and NMDAR-mediated current and/or toxicity have been found to be potentiated in striatal neurons from several HD mouse models as well as heterologous cells expressing the mutant huntingtin protein ${ }^{[10]}$. NMDAR stimulation is also closely linked to mitochondrial activity, because treatment with mitochondrial toxins has been demonstrated to produce striatal damage that can be reversed by the addition of NMDAR antagonists. Future efforts should be focused on the elucidation of molecular pathways linking htt to NMDA receptors, as well as the mechanisms that underlie the enhancement of NMDAR activity by mutant huntingtin.

Intrastriatal infusion of KA can induce excitotoxic lesions in the striatum and has become a well-established chemical model of $\mathrm{HD}^{[71,72]}$. In studies of post-receptor mechanisms of excitotoxicity, our laboratory has found that KA- or the NMDA receptor agonist quinolinic acid (QA)-induced neuronal apoptosis involves activation of NF- $\mathrm{kB}$ and induction of $\mathrm{p} 53$, c-Myc, and other proapoptotic protein ${ }^{[73-75]}$, which seems to mimic HD pathology.

Excitotoxicity in Alzheimer's disease Alzheimer's disease $(\mathrm{AD})$ is a neurodegenerative disorder of the central nervous system associated with progressive cognitive and memory loss. Molecular hallmarks of the disease are extracellular deposition of the $\beta$-amyloid peptide (A $\beta)$ in senile plaques, the appearance of intracellular neurofibrillary tangles (NFT), cholinergic deficit, extensive neuronal loss, and synaptic changes in the cerebral cortex, hippocampus and other areas of brain essential for cognitive and memory functions ${ }^{[76]}$. According to the amyloid cascade hypothesis, $\mathrm{AD}$ pathogenesis is initiated by the overproduction and extracellular deposition of $A \beta$ and the intracellular deposition of NFT. These depositions serve as initiating factors for multiple neurotoxic 
pathways, which may include excitotoxicity, oxidative stress, energy depletion, inflammation and apoptosis ${ }^{[77]}$. Interestingly, recent studies have shown that glutamatergic signaling is compromised by $A \beta$-induced modulation of synaptic glutamate receptors in specific brain regions, paralleling early cognitive deficits $^{[78]}$.

All of these intracellular events converge at the ubiquitous pathways of necrosis and apoptosis. A C-terminal peptide fragment of $A \beta$ can selectively induce neuronal apoptosis in the hippocampal mossy fiber pathway, which might be related to working memory impairment in $\mathrm{AD}^{[79]}$. In addition, there is evidence in $\mathrm{AD}$ that neuronal autophagy is induced but impaired at late steps in the pathway, which may help to explain the intracellular accumulation of $A \beta$ and could contribute to its subsequent extracellular deposition in plaques. Accordingly, $\mathrm{AD}$-causing mutations promote autophagy disruption in the autophagy-lysosomal pathway ${ }^{[80]}$. Microglial cells have also been found in senile plaques, suggesting that damage in the $\mathrm{AD}$ brain could derive from an autoimmune response of microglia that produces free radicals. In addition, oxidative stress and lipid peroxidation have been implicated in glutamate and $A \beta$-induced neurotoxicity ${ }^{[81]}$. A growing body of evidence suggests that perturbations in systems employing the excitatory amino acid $L$-glutamate may underlie the pathogenic mechanisms of chronic neurodegeneration in $\mathrm{AD}^{[82]}$. Under chronic and pathological conditions in $\mathrm{AD}$, loss of mitochondrial membrane potential and the release of apoptotic proteins such as cytochrome $c$, the second mitochondria derived activator of caspase and apoptosis-inducing factor contribute to cell apoptosis $^{[83]}$.

The degeneration caused by $A \beta$ in vivo has also been linked to the excessive activation of NMDA receptors ${ }^{[84]}$. Excitotoxicity resulting from excessive activation of NMDARs may enhance the localized vulnerability of neurons in a manner consistent with $\mathrm{AD}$ neuropathology as a consequence of an altered regional distribution of NMDA receptor subtypes. NMDA receptor antagonists have significant potential for the therapeutic amelioration of $\mathrm{AD}$.

Oxidative stress is another major factor in $\mathrm{AD}$. Researchers have investigated the relationship between oxidative stress and neuronal binding of $A \beta$, which is known to accumulate in the brain tissue of $\mathrm{AD}$ patients. Using hippocampal neuronal cultures,investigator found that $A \beta$ stimulated excessive formation of ROS through a mechanism requiring NMDAR activation $^{[85]}$. Therefore, the dysregulation of NMDAR activity and oxidative stress may have dual deleterious roles in $\mathrm{AD}$.

Excitotoxicity in Parkinson's disease Parkinson's disease (PD) is a neurological disorder that is caused by the degeneration of nigral dopaminergic neurons and the consequent massive drop of dopamine (DA) content in the striatum $^{[86]}$. Despite intense research efforts over many years, the causes of this disease still await elucidation.

Although the complete nature of the complex intra/extracellular signals that regulate this neuronal injury remains to be clarified, a growing body of evidence supports an essential role for oxidative stress in the initiation and progression of the injury process that releases ROS and free radicals and damages cellular proteins, lipids and DNA elements ${ }^{[88]}$. The presynaptic $\alpha$-synuclein is an abundant protein in Lewy bodies, the proteinaceous neuronal inclusions that are the pathological hallmark of sporadic $\mathrm{PD}^{[87]}$. Some studies suggest that nigral neuronal damage in brains of PD patients may release extracellular aggregated $\alpha$-synuclein into the substantia nigra. This may activate microglial ROS production and microglial-enhanced dopaminergic neurodegeneration ${ }^{[89]}$, thereby leading to persistent and progressive nigral neurodegeneration in PD.

The concept of excitotoxicity has also been applied to PD. Studies have demonstrated that parkin (hereditary Parkinson disease PARK2 gene product) regulates the function and stability of excitatory glutamatergic synapses. Postsynaptic expression of parkin dampens excitatory synaptic transmission and causes a marked loss of excitatory synapses in hippocampal neurons. Conversely, knockdown of endogenous parkin or expression of PD-linked parkin mutants profoundly enhances synaptic efficacy and triggers a proliferation of glutamatergic synapses. This proliferation is associated with increased vulnerability to synaptic excitotoxicity ${ }^{[90]}$. The resulting excessive glutamatergic drive could be a source of excitotoxicity in the nigra. As described above, persistent activation of NMDA receptor increases intracellular calcium levels. A role for elevated intracellular calcium in the events leading to cell death in PD is supported by the observation that dopaminergic neurons expressing the calcium-binding protein calbindin may be selectively preserved in $\mathrm{PD}^{[91]}$.

A number of studies have shown that ionotropic antagonists of the NMDA glutamate receptor subtypes counteract parkinsonian symptoms or act in synergy with L-3,4dihydroxyphenylalanine (L-DOPA) in animal models of PD. A key indication that environmental factors may play a role in PD came with the discovery of 1-methyl-4-phenyl-1,2,3,6tetrahydropyridine (MPTP), which has been used to develop animal models of PD in which the pathogenesis of the disease and a variety of therapeutic interventions designed to prevent or reduce dopaminergic neurodegeneration can be studied ${ }^{[92]} . \mathrm{MPP}^{+}$, the active metabolite of MPTP, has been 
shown to inhibit complex I of the mitochondrial electron transport chain, which is thought to be the mechanism by which MPTP induces Parkinsonism. MK-801 (dizocilpine), a noncompetitive antagonist of the NMDA receptor, has also been reported to have protective effects against excitotoxicity induced in dopaminergic systems by MPTP ${ }^{[93]}$. Accumulating evidence suggests that alternative mechanisms of action for MPTP, including release of vesicle-stored dopamine and consequent oxidative damage, release of stored iron deposits, increased cytoplasmic calcium and intraneuronal calcium release ${ }^{[94,95]}$, actually lead to neuronal cell death. Although PD is not typically considered a mitochondrial disease, these studies cited the aforementioned study and other recent work demonstrating that proteins linked to PD affect mitochondrial function or localize to mitochondria ${ }^{[96]}$, suggesting that mitochondrial dysfunction, especially at complex I, may play a larger role in the development of PD than previously realized.

\section{Conclusions and perspectives}

Various mechanisms involving excitotoxicity have been proposed to explain the neuronal cell death characteristic of neurodegenerative diseases, including elevation of intracellular calcium, accumulation of oxidizing free radicals, impairment of mitochondrial function and activation of apoptotic and autophagic programs. Identification of a major postreceptor signaling event that leads to neuronal death could provide a molecular target for therapeutic interventions in many neurodegenerative diseases.

\section{Acknowledgements}

This work was supported by grants from the National Natural Science Foundation of China (№ 30370506; 30772560), Graduate Student Fellowship of Jiangsu Province (№ CX07B_232z), Specialized Research Fund for the Doctoral Program of Higher Education (№ 20050285017) and Soochow University Medical Research Fund (No EE134606).

\section{References}

1 Hague SM, Klaffke S, Bandmann O. Neurodegenerative disorders: Parkinson's disease and Huntington's disease. J Neurol Neurosurg Psychiatry 2005; 76: 1058-63.

2 Javitt DC, Zukin SR. The role of excitatory amino acids in neuropsychiatric illness. J Neuropsychiatry Clin Neurosci 1990; 2: 44-52.

3 Berliocchi L, Bano D, Nicotera P. $\mathrm{Ca}^{2+}$ signals and death programmes in neurons. Philos Trans R Soc Lond B Biol Sci 2005; 360: 2255-8.

4 Wong PC, Cai H, Borchelt DR, Price DL. Genetically engineered mouse models of neurodegenerative diseases. Nat Neurosci 2002; 5: 633-9.

5 Sims NR, Zaidan E. Biochemical changes associated with selective neuronal death following short-term cerebral ischaemia. Int J Biochem Cell Biol 1995; 27: 531-50.

6 Danysz W, Parsons CG. The NMDA receptor antagonist memantine as a symptomatological and neuroprotective treatment for Alzheimer's disease: preclinical evidence. Int J Geriatr Psychiatry 2003; 18: 23-32.

7 Rothstein JD. Excitotoxicity hypothesis. Neurology 1996; 47 (4 Suppl 2): S19-25.

8 Ndountse LT, Chan HM. Role of $N$-methyl- $D$-aspartate receptors in polychlorinated biphenyl mediated neurotoxicity. Toxicol Lett 2009; 184: 50-5.

9 Jung KH, Chu K, Lee ST, Park HK, Kim JH, Kang KM, et al. Augmentation of nitrite therapy in cerebral ischemia by NMDA receptor inhibition. Biochem Biophys Res Commun 2009; 378: 507-12.

10 Fan MMY, Raymond LA. N-Methyl-D-aspartate (NMDA) receptor function and excitotoxicity in Huntington's disease. Neurobiology 2007; 81: 272-93.

11 Handley OJ, Naji JJ, Dunnett SB, Rosser AE. Pharmaceutical, cellular and genetic therapies for Huntington's disease. Clin Sci 2006; 110: 73-88.

12 Van Damme P, Bogaert E, Dewil M, Hersmus N, Kiraly D, Scheveneels W, et al. Astrocytes regulate GluR2 expression in motor neurons and their vulnerability to excitotoxicity. Proc Natl Acad Sci USA 2007; 104: 14825-30.

13 Wu HY, Tomizawa K, Oda Y. Critical role of calpain-mediated cleavage of calcineurin in excitotoxic neurodegeneration. Biol Chem 2004; 279: 4929-40.

14 Wang Y, Han R, Liang ZQ, Wu JC, Zhang XD, Gu ZL, et al. An autophagic mechanism is involved in apoptotic death of rat striatal neurons induced by the non- $N$-methyl- $D$-aspartate receptor agonist kainic acid. Autophagy 2008; 4: 214-26.

15 Wang Y, Gu ZL, Cao Y, Liang ZQ, Han R, Bennett MC, et al. Lysosomal enzyme cathepsin B is involved in kainic acid-induced excitotoxicity in rat striatum. Brain Res 2006; 1071: 245-9.

16 Friedman LK. Calcium: a role for neuroprotection and sustained adaptation. Mol Interv 2006; 6: 315-29.

17 Lee HG, Zhu X, Casadesus G, Pallàs M, Camins A, O’Neill MJ, et al. The effect of mGluR2 activation on signal transduction pathways and neuronal cell survival. Brain Res 2009; 1249: 244-50.

18 Yadava N, Nicholls DG. Spare respiratory capacity rather than oxidative stress regulates glutamate excitotoxicity after partial respiratory inhibition of mitochondrial complex i with rotenone. J Neurosci 2007; 27: 7310-7.

19 Qin ZH, Tao LY, Chen X. Dual roles of NF-kB in cell survival and implications of NF- $\mathrm{kB}$ inhibitors in neuroprotective therapy. Acta Pharmacol Sin 2007; 28: 1859-72.

20 Beck J, Lenart B, Kintner DB, Sun D. Na-K-Cl cotransporter contributes to glutamate-mediated excitotoxicity. J Neurosci 2003; 23: 5061-8.

21 Chen Q, Olney JW, Lukasiewicz PD, Almli T, Romano C. $\mathrm{Ca}^{2+}$ independent excitotoxic neurodegeneration in isolated retina, an 
intact neural net: a role for $\mathrm{Cl}^{-}$and inhibitory transmitters. Mol Pharmacol 1998; 53: 564-72.

22 Olney JW, Price MT, Samson L, Labruyere J. The role of specific ions in glutamate neurotoxicity. Neurosci Lett 1986; 65: 65-71.

23 Inglefield JR, Schwartz-Bloom RD. Optical imaging of hippocampal neurons with a chloride-sensitive dye: early effects of in vitro ischemia. J Neurochem 1998; 70: 2500-9.

24 Staub F, Stoffel M, Berger S, Eriskat J, Baethmann A. Treatment of vasogenic brain edema with the novel $\mathrm{Cl}^{-}$transport inhibitor torasemide. J Neurotrauma 1994; 11: 679-90.

25 Lee BK, Lee DH, Park S, Park SL, Yoon JS, Lee MG, et al. Effects of KR-33028, a novel $\mathrm{Na}^{+} / \mathrm{H}^{+}$exchanger-1 inhibitor, on glutamateinduced neuronal cell death and ischemia-induced cerebral infarct. Brain Res 2009; 1248: 22-30.

26 Wang HG, Pathan N, Ethell IM, Kjrajewski S, Yamaguchi Y, Shibasaki F, et al. $\mathrm{Ca}^{2+}$ induced apoptosis through calcineurin dephosphorylation of BAD. Science 1999; 284: 339-43.

27 Youn HD, Sun L, Prywes R, Liu JO. Apoptosis of T cells mediated by $\mathrm{Ca}^{2+}$-induced release of the transcription factor MEF2. Science 1999; 286: 790-3.

28 Szado T, Vanderheyden V, Parys JB, De Smedt H, Rietdorf K, Kotelevets L, et al. Phosphorylation of inositol 1,4,5-trisphosphate receptors by protein kinase $\mathrm{B} / \mathrm{Akt}$ inhibits $\mathrm{Ca}^{2+}$ release and apoptosis. Proc Natl Acad Sci USA 2008; 105: 2427-32.

29 Nixon RA. A "Protease Activation Cascade" in the Pathogenesis of Alzheimer's Disease. Ann NY Acad Sci 2000; 924: 117-31.

30 Wang KK. Calpain and caspase: can you tell the difference? Trends Neurosci 2000; 23: 20-6.

31 Ichinose T, Yu S, Wang XQ, Yu SP. $\mathrm{Ca}^{2+}$-independent, but voltage and activity-dependent regulation of the NMDA receptor outward $\mathrm{K}^{+}$current in mouse cortical neurons. J Physiol 2003; 551: 40317.

32 Nicholls DG. Mitochondrial dysfunction and glutamate excitotoxicity studied in primary neuronal cultures. Curr Mol Med 2004; 4: 149-77.

33 Farooqui T, Farooqui AA. Aging: an important factor for the pathogenesis of neurodegenerative diseases. Mech Ageing Dev 2009; 130: 203-15.

34 Albers DS, Swerdlow RH, Manfredi G, Gajewski C, Yang L, Parker WD, et al. Further evidence for mitochondrial dysfunction in progressive supranuclear palsy. Exp Neurol 2001; 168: 196-8.

35 Gilgun-Sherki Y, Rosenbaum Z, Melamed E, Offen D. Antioxidant therapy in acute central nervous system injury: current state. Pharmacol Rev 2002; 54: 271-84.

36 Porta S, Serra SA, Huch M, Valverde MA, Llorens F, Estivill X, et al. RCAN1 (DSCR1) increases neuronal susceptibility to oxidative stress: a potential pathogenic process in neurodegeneration. Hum Mol Genet 2007; 16: 1039-50.

37 Fridovich I. Superoxide anion radical $\left(\mathrm{O}_{2}{ }^{-}\right)$, superoxide dismutases, and related matters. J Biol Chem 1997; 272: 18515-7.

38 Law A, Gauthier S, Quirion R. Say NO to Alzheimer's disease, the putative links between nitric oxide and dementia of Alzheimer's type. Brain Res Rev 2001; 35: 73-96.

39 Brown G, Bal-Price A. Inflammatory neurodegeneration mediated by nitric oxide, glutamate and mitochondria. Mol Neurobiol 2003; 27: 325-55.

40 Parathath SR, Parathath S, Tsirka SE. Nitric oxide mediates neurodegeneration and breakdown of the blood-brain barrier in
tPA-dependent excitotoxic injury in mice. J Cell Sci 2005; 119: 339-49.

41 Wei C, Qin ZH, Zhang HL. Matrix metalloproteinases and nervous diseases. Chin Pharmacol Bull 2007; 23: 8-12.

42 Berman SB, Hastings TG. Dopamine oxidation alters mitochondrial respiration and induces permeability transition in brain mitochondria, implications for Parkinson's disease. J Neurochem 1999; 73: 1127-37.

43 Halestrap AP, Doran E, Gillespie JP, O’Toole A. Mitochondria and cell death. Biochem Soc Trans 2000; 28: 170-7.

44 Tournier C, Hess P, Yang DD, Xu J, Turner TK, Nimnual A, et al. Requirement of JNK for stress-induced activation of the cytochrome c-mediated death pathway. Science 2000; 288: 870-4.

45 Luo Y, Hattori A, Munoz J, Qin ZH, Roth GS. Intrastriatal dopamine injection induces apoptosis through oxidation-involved activation of transcription factors AP-1 and NF-kappaB in rats. Mol Pharmacol 1999; 56: 254-64.

46 Maalouf M, Sullivan PG, Davis L, Kim DY, Rho JM. Ketones inhibit mitochondrial production of reactive oxygen species production following glutamate excitotoxicity by increasing $\mathrm{NADH}$ oxidation. Neuroscience 2007; 145: 256-64.

47 Morgan MJ, Kim YS, Liu ZG. Lipid rafts and oxidative stressinduced cell death. Antioxidants Redox Signal 2007; 9: 1471-84.

48 Duchen MR. Roles of mitochondria in health and disease. Diabetes 2004; 53: 96-102.

49 Orrenius S. Mitochondrial regulation of apoptotic cell death. Toxicol Lett 2004; 149: 19-23.

50 Yan CH, Liang ZQ, Gu ZL, Yang YP, Reid P, Qin ZH. Contributions of autophagic and apoptotic mechanisms to CrTX-induced death of K562 cells. Toxicon 2006; 47: 521-30.

51 Kim I, Rodriguez-Enriquez S, Lemasters JJ. Selective degradation of mitochondria by mitophagy. Arch Biochem Biophys 2007; 462: 245-53.

52 Kazuhiko N, Osamu Y, Kinya O. Crosstalk between autophagy and apoptosis in heart disease. Circ Res 2008; 103: 343-51.

53 Qin ZH, Wang Y, Nakai M, Chase TN. Activation of transcription factor NF- $\mathrm{kB}$ contributes to excitotoxin-induced apoptosis. Mol Pharmacol 1998; 53: 33-42.

54 Qin ZH, Wang Y, Nakai M, Chen RW, Chuang DM, Chase TN. Co-stimulation of cyclic-AMP-linked metabotropic glutamate receptors in rat striatum attenuates excitotoxin-induced nuclear factor- $\kappa \mathrm{B}$ activation and apoptosis. Neuroscience 1999; 94: 115362.

55 Kessler DJ, Duyao MP, Spicer DB, Sonenshei GE. NF-кB-like factors mediate interleukin 1 induction of $\mathrm{c}-\mathrm{Myc}$ gene transcription in fibroblasts. J Exp Med 1992; 176: 787-92.

$56 \mathrm{Wu} \mathrm{H}$, Lozano G. NF- $\mathrm{kB}$ activation of $\mathrm{p} 53$, a potential mechanism for suppressing cell growth in response to stress. J Biol Chem 1994; 269: 20067-74.

57 Lee H, Arsura M, Wu M, Duyao M, Buckler AJ, Sonenshein GE. Role of Rel-related factors in control of c-Myc gene transcription in receptor-mediated apoptosis of marine B cell WEHI 231 line. J Exp Med 1995; 181: 1169-77.

58 O'Connor JC, Wallace DM, O’Brien CJ, Cotter TG. A novel antioxidant function for the tumor-suppressor gene p53 in the retinal ganglion cell. Investig Ophthalmol Visual Sci 2008; 49: 4237-44.

59 Yuan J, Yankner BA. Apoptosis in the nervous system. Nature 
2000 ; 407: 802-9.

60 Navarro A, Boveris A. The mitochondrial energy transduction system and the aging process. Am J Physiol Cell Physiol 2007; 292: 670-86.

61 Vonsattel JP, DiFiglia M. Huntington disease. J Neuropathol Exp Neurol 1998; 57: 369-84.

62 Deng P, Zhang Y, Xu ZC. Inhibition of $I_{\mathrm{h}}$ in striatal cholinergic interneurons early after transient forebrain ischemia. J Cereb Blood Flow Metab 2008; 28: 939-47.

63 Li Y, Lei ZG, Xu ZC. Enhancement of inhibitory synaptic transmission in large aspiny neurons after transient cerebral ischemia. Neuroscience 2009; Jan 3. In Press.

64 Van Raamsdonk JM, Pearson J, Rogers DA, Bissada N, Vogl AW, Hayden MR, et al. Loss of wild-type huntingtin influences motor dysfunction and survival in the YAC128 mouse model of Huntington disease. Hum Mol Genet 2005; 14: 1379-92.

65 Zhang H, Li Q, Graham RK, Slow E, Hayden MR, Bezprozvanny I. Full length mutant huntingtin is required for altered $\mathrm{Ca}^{2+}$ signaling and apoptosis of striatal neurons in the YAC mouse model of Huntington's disease. Neurobiol Dis 2008; 31: 80-8.

66 Song C, Zhang Y, Parsons CG, Liu YF. Expression of polyglutamine-expanded huntingtin induces tyrosine phosphorylation of N-methyl-D-aspartate receptors. J Biol Chem 2003; 278: 33364-9.

67 Roche KW, Standley S, McCallum J, Dune Ly C, Ehlers MD, Wenthold RJ. Molecular determinants of NMDA receptor internalization. Nat Neurosci 2001; 4: 794-802.

68 Sun Y, Savanenin A, Reddy PH, Liu YF. Polyglutamine-expanded huntingtin promotes sensitization of $N$-methyl- $D$-aspartate receptors via post-synaptic density 95. J Biol Chem 2001; 276: 24713-8.

69 Zeron MM, Chen N, Moshaver A, Lee AT, Wellington CL, Hayden $\mathrm{MR}$, et al. Mutant huntingtin enhances excitotoxic cell death. Mol Cell Neurosci 2001; 17: 41-53.

70 Liang ZQ, Wang XX, Wang YM, Chang DM, DiFiglia M, Chase $\mathrm{TN}$, et al. Susceptibility of striatal neurons to excitotoxic injury correlates with basal levels of bcl-2 and the induction of p53 and c-myc immunoreactivity. Neurobiol Dis 2005; 20: 562-73.

71 Coyle JT, Schwarcz R. Lesion of striatal neurones with kainic acid provides a model for Huntington's chorea. Nature 1976; 263: 244-6.

72 McGeer EG, McGeer PL. Duplication of biochemical changes of Huntington's chorea by intrastriatal injections of glutamic and kainic acids. Nature 1976; 63: 517-9.

73 Nakai M, Qin ZH, Chen JF, Wang Y, Chase TN. Kainic acidinduced apoptosis in rat striatum is associated with nuclear factor$\kappa B$ activation. J Neurochem 2000; 74: 647-58.

74 Liang ZQ, Wang XX, Wang Y, Chen RW, Chuang DM, Chase $\mathrm{TN}$, et al. NF-kB-dependent cyclin D1 induction associated with $N$-methyl- $D$-aspartate receptor-mediated apoptosis in rat striatum. J Neurosci Res 2007; 85: 1295-309.

75 Qin ZH, Chen RW, Wang Y, Nakai M, Chuang DM, Chase TN. NF- $\kappa B$ nuclear translocation up-regulates c-Myc and p53 during $\mathrm{N}$-methyl- $D$-aspartate receptor-mediatedapoptosis. J Neurosci 1999; 19: 4023-33.

76 Busciglio J, Yankner BA. Apoptosis and increased generation of reactive oxygen species in Down's syndrome neurons in vitro. Nature 1995; 378: 776-9.

77 Robinson SR, Bishop GM. Aß as a bioflocculant: implications for the amyloid hypothesis of Alzheimer's disease. Neurobiol Aging 2002; 23 : 1051-72.

78 Parameshwaran K, Dhanasekaran M, Suppiramaniam V. Amyloid beta peptides and glutamatergic synaptic dysregulation. Exp Neurol 2008; 210: 7-13.

79 Jinno S, Araki K, Matsumoto Y, Suh YH, Yamamoto T. Selective apoptosis induction in the hippocampal mossy fiber pathway by exposure to CT105, the C-terminal fragment of Alzheimer's amyloid precursor protein. Brain Res 2009; 1249: 68-78.

80 Nixon RA. Autophagy, amyloidogenesis and Alzheimer disease. J Cell Sci 2007; 120: 4081-91.

81 Parihar MS, Hemnani T. Alzheimer's disease pathogenesis and therapeutic Intervent. J Clin Neurosci 2004; 11: 456-67.

82 Hynd MR, Scott HL, Dodd PR. Glutamate mediated excitotoxicity and neurodegeneration in Alzheimer's disease. Neurochem 2004; 45: 583-95.

83 Kuo CT, Hsua MJ, Chen BC, Chen CC, Teng CM, Pan SL, et al. Denbinobin induces apoptosis in human lung adenocarcinoma cells via Akt inactivation, Bad activation, and mitochondrial dysfunction. Toxicol Lett 2008; 177: 48-58.

84 Miguel-Hidalgo JJ, Alvarez XA, Cacabelos R, Quack G. Neuroprotection by memantine against neurodegeneration induced by

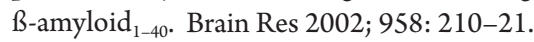

85 De Felice FG, Velasco PT, Lambert MP, Viola K, Fernandez SJ, Ferreira ST, et al. Aß oligomers induce neuronal oxidative stress through an $N$-methyl- $D$-aspartate receptor-dependent mechanism that is blocked by the alzheimer drug memantine. J Biol Chem 2007; 282: 11590-601.

86 Obeso JA, Olanou CW, Nutt JG. Levodopa motor complications in Parkinson's disease. Trends Neurosci 2000; 23: 2-7.

87 Mouradian MM. Recent advances in the genetics and pathogenesis of Parkinson disease. Neurology 2002; 58: 179-85.

88 Jenner P. Oxidative mechanisms in nigral cell death in Parkinson's disease. Mov Disord 1998; 13: 24-34.

89 Zhang W, Wang TG, Pei Z, Miller DS, Wu XF, Block ML, et al. Aggregated-synuclein activates microglia: a process leading to disease progression in Parkinson's disease. FASEB J 2005; 19: 533-42.

90 Helton TD, Otsuka T, Lee MC, Mu YY, Ehlers MD. Pruning and loss of excitatory synapses by the Parkin ubiquitin ligase. Proc Natl Acad Sci USA 2008; 105: 19492-7.

91 Lang AE, Lozano AM. Parkinson's disease-first of two parts. Med Prog 1998; 339: 1044-53.

92 Dauer W, Przedborski S. Parkinson's disease: mechanisms and models. Neuron 2003; 39: 889-909.

93 Moring J, Niego LA, Ganley LM, Trumbore MW, Herbette LG. Interaction of the NMDA receptor noncompetitive antagonist mk-801 with model and native membranes. Biophys J 1994; 67: 2376-86.

94 Kooncumchoo P, Sharma S, Porter J, Govitrapong P, Ebadi M. Coenzyme Q (10) provides neuroprotection in iron-induced apoptosis in dopamine neurons. J Mol Neurosci 2006; 28: 125-41.

95 Obata T. Nitric oxide and MPP (t)-induced hydroxyl radical generation. J Neural Transm 2006; 113: 1131-44.

96 Aleyasin H, Rousseaux MW, Phillips M, Kim RH, Bland RJ, Callaghan S, et al. The Parkinson's disease gene DJ-1 is also a key regulator of stroke-induced damage. Proc Natl Acad Sci USA 2007; 104: 18748-53. 